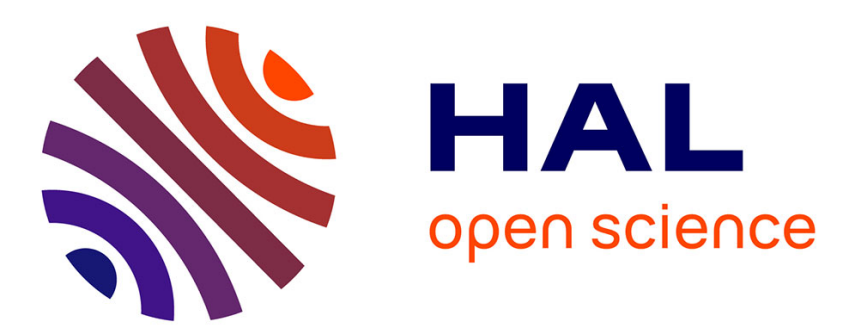

\title{
Polynomes de Tchebytchev et modes de transmission totale dans les multicouches périodiques
}

\author{
Ph. Gatignol, J. Moukemaha
}

\section{To cite this version:}

Ph. Gatignol, J. Moukemaha. Polynomes de Tchebytchev et modes de transmission totale dans les multicouches périodiques. Journal de Physique IV Proceedings, 1994, 04 (C5), pp.C5-817-C5-820. 10.1051/jp4:19945176 . jpa-00252857

\section{HAL Id: jpa-00252857 https://hal.science/jpa-00252857}

Submitted on 1 Jan 1994

HAL is a multi-disciplinary open access archive for the deposit and dissemination of scientific research documents, whether they are published or not. The documents may come from teaching and research institutions in France or abroad, or from public or private research centers.
L'archive ouverte pluridisciplinaire HAL, est destinée au dépôt et à la diffusion de documents scientifiques de niveau recherche, publiés ou non, émanant des établissements d'enseignement et de recherche français ou étrangers, des laboratoires publics ou privés. 


\title{
Polynomes de Tchebytchev et modes de transmission totale dans les multicouches périodiques
}

\author{
Ph. GATIGNOL et J.S. MOUKEMAHA
}

\begin{abstract}
Laboratoire de Génie Mécanique pour les Matériaux et les Structures, URA 1505 du CNRS, Université de Technologie de Compiègne, BP. 649, 60206 Compiègne cedex, France
\end{abstract}

\begin{abstract}
The acoustic wave propagation in periodically layered media has been successfully described by Floquet theory during these last years. However, this method requires the determination of eigenvalues of the period transfer matrix, which may cost time for numerical developments. Moreover, a number of numerical (and experimental) observations, such as the existence and frequency distribution of total transmission modes, cannot be explained through the Floquet formalism. Here we propose an alternative approach, based on matricial algebraic properties, which enables to express the transfer matrix of the whole structure in terms of the period matrix and its trace as an argument of Tchebytchev polynomials of the second kind. Besides numerical facilities introduced by this new algorithm, the analytic form of the transfer matrix so obtained gives the key for the understanding of the distribution of eigenvibrations and total transmission modes of the N-periodic structure. As a main result, two families of modes are shown to be present: the first one is associated with the basic period, the other depends on the whole structure.
\end{abstract}

\section{1 - Introduction et présentation du modèle}

L'étude non destructive des matériaux composites par les techniques ultrasonores a suscité, depuis une dizaine d'années, un intérêt pour la propagation du son dans les milieux périodiques. Les modèles les plus simples sont constitués d'une juxtaposition périodique de couches élastiques homogènes (voir [1] par exemple). Pour de tels milieux, le concept d'ondes de Floquet s'est révélé fructueux ([2],[3]). Il permet de décrire, de manière simple, le champ acoustique dans la structure et son introduction est nécessaire si l'on souhaite exprimer les conditions de rayonnement à l'infini dans le cas de structures non bornées. Ce concept a été étendu sans difficultés au cas où les couches constitutives sont anisotropes ([4],[5]).

Par ailleurs, les modèles périodiques unidimensionnels ont permis, grâce à leur simplicité, d'introduire des défauts (porosités ou délaminages) dans la modélisation ([6],[7]). La compréhension des phénomènes passe par une connaissance complète du comportement acoustique des structures saines, constituées d'un nombre fini de périodes. Pour de telles structures, on a observé numériquement et expérimentalement ([8]) l'existence de modes de transmission totale.

Le but du présent travail est de proposer, pour les modèles unidimensionnels, une alternative à la méthode des ondes de Floquet. Cette nouvelle méthode, qui utilise les propriétés algébriques des matrices de transfert, permet une description analytique plus simple du champ acoustique (et plus rapide sur le plan numérique) et conduit à une explication claire de l'existence des modes de transmission totale. 
Le modèle physique étudié est décrit sur la Figure, avec les notations employées. Les milieux sont supposés isotropes. La propagation étant normale aux interfaces, seules les ondes longitudinales sont concernées, de telle sorte qu'on peut considérer que les milieux ont un comportement de type fluide. Le motif (ou période) de base $\boldsymbol{P}$ est constitué d'un certain nombre de couches d'impédances $Z_{\mathrm{i}}$, de célérités du son $c_{i}$, d'épaisseurs $h_{i}$. La structure complète est formée par la juxtaposition de $N$ périodes. Elle sépare deux fluides extrêmes d'extension infinie d'impédances $Z_{0}$ et $Z_{\mathrm{f}}$.

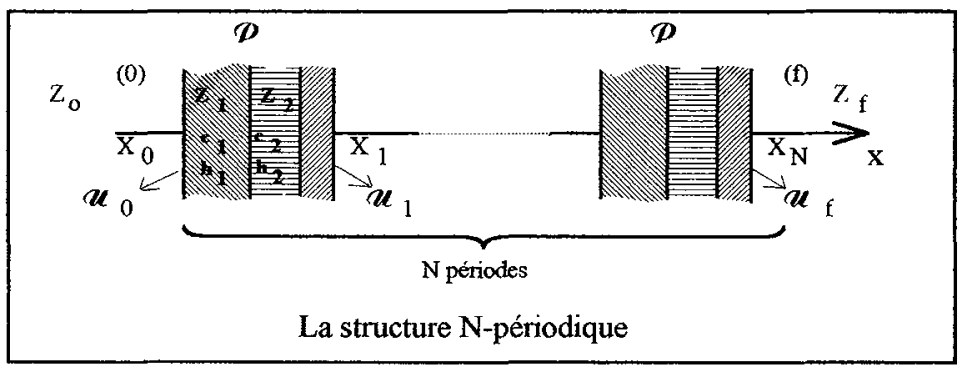

\section{2 - Les variables physiques et le formalisme matriciel}

Les ondes sont supposées monochromatiques (dépendance temporelle: $\exp (-\mathrm{i} \omega t)$ ). Le champ acoustique, dans chaque milieu, est décrit par la vitesse particulaire $u$ (dans la direction $\mathrm{x}$ ) et par la pression p . La continuité de ces deux grandeurs à la traversée de chaque interface permet de spécifier l'état acoustique de la structure en chacune de ses interfaces par la matrice d'état:

$$
\boldsymbol{u}=\left(\begin{array}{l}
\mathrm{u} \\
\mathrm{p}
\end{array}\right)
$$

La méthode des matrices de transfert permet de relier entre elles les matrices d'état aux diverses interfaces, et finalement d'exprimer la matrice d'état $\boldsymbol{u}_{\mathrm{f}}$ à l'interface de sortie $\mathrm{x}=\mathrm{X}_{\mathrm{N}}$ en fonction de la matrice d'état $\boldsymbol{u}_{0}$ à l'interface d'entrée $\mathrm{x}=\mathrm{X}_{0}$.

Dans le cas d'une seule couche, homogène isotrope, la matrice de transfert s'écrit:

$$
\boldsymbol{g}=\left(\begin{array}{cc}
\cos \omega T & (\mathrm{i} / \mathrm{Z}) \sin \omega T \\
\mathrm{i} Z \sin \omega \mathrm{T} & \cos \omega \mathrm{T}
\end{array}\right) \quad \text { avec: } \quad \mathrm{T}=\frac{\mathrm{h}}{\mathrm{c}}
$$

Cette matrice est de la forme:

$$
\boldsymbol{g}=\left(\begin{array}{cc}
\mathrm{a}(\omega) & \mathrm{ib}(\omega) \\
\mathrm{ic}(\omega) & \mathrm{d}(\omega)
\end{array}\right)
$$

où $\mathrm{a}, \mathrm{b}, \mathrm{c}, \mathrm{d}$ sont des fonctions formellement réelles de $\omega$, vérifiant la relation:

$$
\operatorname{det} \boldsymbol{\nabla}=\mathrm{ad}+\mathrm{bc}=1
$$

et telles que a et $d$ soient paires, tandis que $b$ et $\mathrm{c}$ sont impaires.

Les matrices d'ordre 2 vérifiant l'ensemble de ces propriétés constituent un groupe multiplicatif. En particulier, la matrice de période, produit d'un nombre de matrices de cette forme égal au nombre de couches constituant la période de base, s'écrit: 


$$
\boldsymbol{g}_{\mathfrak{D}}=\left(\begin{array}{cc}
\mathrm{a}_{\boldsymbol{p}}(\omega) & \mathrm{i} \mathrm{b}_{\boldsymbol{p}}(\omega) \\
\mathrm{i} \mathrm{c}_{\mathcal{P}}(\omega) & \mathrm{d}_{\mathcal{P}}(\omega)
\end{array}\right)
$$

avec les propriétés précédentes pour les fonctions $\mathrm{a}_{\mathfrak{D}}, \ldots$.

On rappelle (voir en particulier [2]) que les ondes de Floquet sont liées aux valeurs et vecteurs propres de la matrice $\boldsymbol{\partial}_{\mathfrak{P}}$. Si la somme des valeurs propres (dont le produit est égal à 1 d'après (4)) :

$$
\mathrm{s}_{\mathcal{D}}(\omega)=\mathrm{a}_{\mathcal{P}}(\omega)+\mathrm{d}_{\mathcal{D}}(\omega)
$$

est supérieure à 2 en valeur absolue, ces valeurs propres sont réelles et les ondes de Floquet sont de type évanescent. Les valeurs de la fréquence pour lesquelles il en est ainsi constituent les bandes d'arrêt, séparées entre elles par des bandes passantes pour lesquelles les ondes de Floquet sont propagatives.

\section{3 - Structure complète, matrice de structure et méthode de calcul}

Dans le cas de la structure complète, constituée de $\mathrm{N}$ périodes $\boldsymbol{P}$, la matrice de transfert reliant les vecteurs d'état d'entrée et de sortie:

$$
\boldsymbol{u}_{\mathrm{f}}=\boldsymbol{\partial}_{\mathrm{NP}} \boldsymbol{u}_{0}
$$

est la puissance $\mathrm{N}$-ième de la matrice de période $\boldsymbol{J}_{\mathfrak{D}}$. Elle est de la forme indiquée (3),(4) . Le théorème de Cayley-Hamilton et la propriété (4) permettent d'exprimer cette matrice sous la forme suivante:

$$
\boldsymbol{\partial}_{\mathrm{N} \boldsymbol{p}}=\left\{\boldsymbol{\partial}_{\boldsymbol{p}}\right\}^{\mathrm{N}}=\Sigma_{\mathrm{N}-1}\left(\mathrm{~s}_{\boldsymbol{p}}\right) \boldsymbol{\partial}_{\boldsymbol{p}}-\Sigma_{\mathrm{N}-2}\left(\mathrm{~s}_{\boldsymbol{p}}\right) \boldsymbol{Z}
$$

où $\mathcal{Z}$ est la matrice unité d'ordre 2. Les $\Sigma_{\mathrm{n}}$ (s) sont les polynômes définis par les relations de récurrence :

$$
\Sigma_{n}=s \Sigma_{n-1}-\Sigma_{n-2}, \Sigma_{0}=1, \Sigma_{1}=\mathrm{s}
$$

Ils peuvent s'exprimer à l'aide des polynômes de Tchebytchev de seconde espèce $U_{n}(x)$ sous la forme:

$$
\Sigma_{\mathrm{n}}(\mathrm{s})=\mathrm{U}_{\mathrm{n}}(\mathrm{s} / 2)
$$

Les éléments de la matrice de structure $\boldsymbol{\partial}_{\mathrm{N} P}$ s'expriment donc en fonction de ceux de la matrice de période $\boldsymbol{g}_{\mathfrak{D}}$ suivant les relations:

$$
\begin{aligned}
& \mathrm{a}_{\mathrm{N} \boldsymbol{p}}=\mathrm{a}_{\boldsymbol{p}} \Sigma_{\mathrm{N}-1}\left(\mathrm{~s}_{\boldsymbol{p}}\right)-\Sigma_{\mathrm{N}-2}\left(\mathrm{~s}_{\boldsymbol{p}}\right), \quad \mathrm{b}_{\mathrm{N} \boldsymbol{p}}=\mathrm{b}_{\boldsymbol{p}} \Sigma_{\mathrm{N}-1}\left(\mathrm{~s}_{\boldsymbol{p}}\right) \\
& \mathrm{c}_{\mathrm{N} \mathcal{P}}=\mathrm{c}_{\mathfrak{P}} \Sigma_{\mathrm{N}-1}\left(\mathrm{~s}_{\mathfrak{p}}\right), \mathrm{d}_{\mathrm{N} \mathcal{D}}=\mathrm{d}_{\mathfrak{p}} \Sigma_{\mathrm{N}-1}\left(\mathrm{~s}_{\boldsymbol{P}}\right)-\Sigma_{\mathrm{N}-2}\left(\mathrm{~s}_{\mathfrak{P}}\right)
\end{aligned}
$$

\section{4 - Vibrations propres et modes de transmission totale}

Les vibrations propres de la structure $\mathrm{N}$-périodique placée dans le vide s'obtiennent par les conditions $\mathrm{p}_{0}=\mathrm{p}_{\mathrm{f}}=0$, qui conduisent à l'équation aux fréquences propres:

$$
\mathbf{c}_{\mathrm{N} \boldsymbol{\mathcal { P }}}(\omega)=0
$$

D'après (11), cette équation se factorise, ce qui conduit à deux familles de vibrations propres:

- les vibrations propres liées à la période, solutions de:

$$
c_{\mathscr{p}}(\omega)=0
$$

En vertu de la relation (4), les fréquences correspondantes sont nécessairement situées dans les bandes d'arrêt.

- les vibrations propres liées à la structure $\mathrm{N}$-périodique, solutions de:

$$
\Sigma_{N-1}\left[s_{\mathscr{P}}(\omega)\right]=0
$$


D'après les propriétés des zéros des polynômes de Tchebytchev, $\Sigma_{\mathrm{n}}(\mathrm{s})$ a tous ses zéros réels, simples et situés dans lintervalle $]-2,+2[$. Les solutions de (14) sont donc toutes situées à l'intérieur des bandes passantes.

Les modes de transmission totale à travers la structure $\mathrm{N}$-périodique, du milieu $(0)$ vers le milieu (f), s'obtiennent en écrivant les deux conditions d'impédance:

$$
\mathrm{p}_{0}=\mathrm{Z}_{0} \mathrm{u}_{0} \quad, \quad \mathrm{p}_{\mathrm{f}}=\mathrm{Z}_{\mathrm{f}} \mathrm{u}_{\mathrm{f}}
$$

L'équation aux fréquences correspondante s'écrit, dans le cas particulier oủ les impédances extrèmes sont égales $\left(Z_{\mathrm{f}}=Z_{0}\right)$ :

$$
Z_{0}\left(a_{N \boldsymbol{p}}-d_{N \boldsymbol{p}}\right)+\mathrm{i}\left(Z_{0}^{2} b_{N \boldsymbol{p}}-c_{N \boldsymbol{p}}\right)=0
$$

D'après les relations (11), cette équation se factorise sous la forme:

$$
\left[\mathrm{Z}_{0}\left(\mathrm{a}_{\boldsymbol{p}}-\mathrm{d}_{\boldsymbol{p}}\right)+\mathrm{i}\left(\mathrm{Z}_{0}^{2} \mathrm{~b}_{\boldsymbol{D}}-\mathrm{c}_{\boldsymbol{D}}\right)\right] \Sigma_{\mathrm{N}-1}\left(\mathrm{~s}_{\boldsymbol{D}}\right)=0
$$

On obtient ainsi deux familles de transmissions totales: 1/ les transmissions totales liées à la période, dont les fréquences annulent le premier facteur de l'équation (17). Ces solutions sont en général complexes, mais proches des bandes d'arrêt définies sur l'axe réel. 2/ les transmissions totales liées à la structure $\mathrm{N}$ périodique dont les fréquences sont réelles et s'identifient à celles des vibrations propres, solutions de l'équation (14).

Dans le cas de la période à deux couches constitutives, les fonctions de la fréquence:

$$
\begin{aligned}
& \mathrm{s}_{\mathcal{D}}(\omega)=2 \cos \left(\omega \mathrm{T}_{1}\right) \cos \left(\omega \mathrm{T}_{2}\right)-\frac{Z_{1}^{2}+Z_{2}^{2}}{Z_{1} Z_{2}} \sin \left(\omega \mathrm{T}_{1}\right) \sin \left(\omega \mathrm{T}_{2}\right) \\
& \mathrm{c}_{\boldsymbol{P}}(\omega)=Z_{1} \sin \left(\omega \mathrm{T}_{1}\right) \cos \left(\omega \mathrm{T}_{2}\right)+Z_{2} \cos \left(\omega \mathrm{T}_{1}\right) \sin \left(\omega \mathrm{T}_{2}\right)
\end{aligned}
$$

sont telles que la première n'ait d'extremum que dans les bandes d'arrêt et que ses zéros s'intercalent avec ceux de la seconde. On en déduit qu'il existe une et une seule vibration propre de période par bande d'arrêt et qu'une structure constituée de $\mathrm{N}$ périodes possède exactement $\mathrm{N}-1$ vibrations propres (et transmissions totales) dans chaque bande passante.

\section{5 - Conclusions}

La méthode proposée permet d'accéder aisément aux transmissions totales du multicouche. Elle permet également d'exprimer le coefficient de réflexion et d'étudier les résonances. Elle s'étend sans peine à des configurations plus complexes.

\section{Références:}

[1] Sha A.H.and Datta S.K., Int.J.Solids Structures 18/5 (1982) 397-410.

[2] Rousseau M. et Gatignol Ph., Acustica 64/4 (1987) 169-228.

[3] Rousseau M., J. Ac. Soc.America 86 (1989) 2369-2376.

[4] Braga A.M.B. and Herrmann G., J. Ac.Soc.America 91/3 (1992) 1211-1227.

[5] Potel C. and de Belleval J.F.,J. Ac.Soc.America 93/5 (1993) 2669-2677.

[6] Moukemaha J.S., Gatignol Ph. et de Belleval J.F.,"Modélisation unidimensionnelle pour la détection par ultrasons des défauts dans un multicouche périodique", Premier Congrès Français d'Acoustique Volume I, P. Filippi et M.Zakharia Ed., Lyon France avril 1990 (Les Editions de Physique, Les Ulis, 1990) pp.451-454.

[7] Moukemaha J.S.,"Modélisation des signaux acoustiques réfléchis par un multicouche périodique. Application à l'identification de défauts par méthodes ultrasonores", Thèse de Doctorat de l'Université de Technologie de Compiègne, mars 1991.

[8] Khelil M., Izbicki J.L., Lenoir O., Rousseau M. et Gatignol Ph., "Experimental determination of periodically structure resonances; interpretation by Floquet theory", Europ. Conf. on Underwater Acoustics, N. Weydert Ed., Luxembourg septembre 1992. 